This item was submitted to Loughborough's Institutional Repository (https://dspace.lboro.ac.uk/) by the author and is made available under the following Creative Commons Licence conditions.

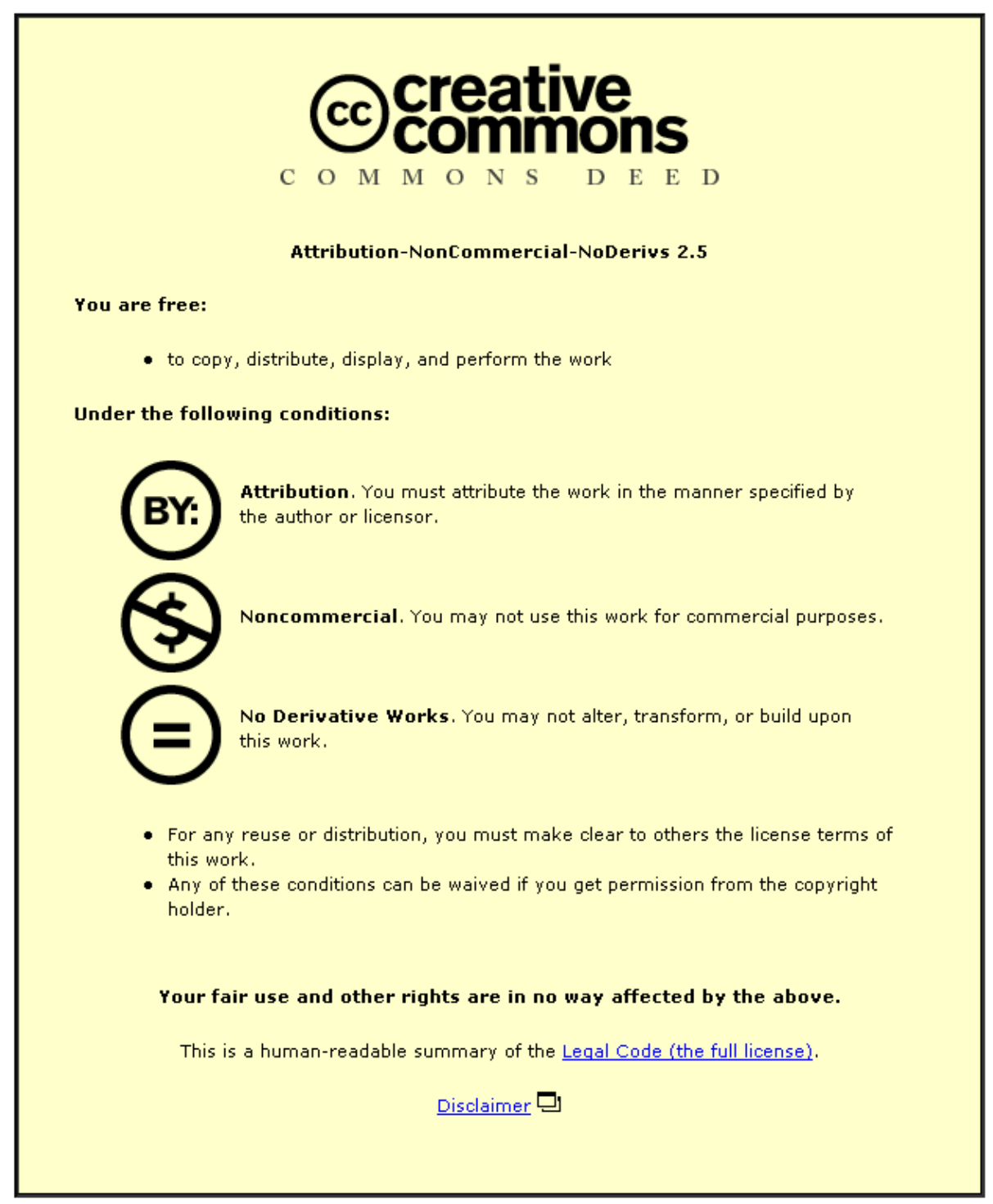

For the full text of this licence, please go to: http://creativecommons.org/licenses/by-nc-nd/2.5/ 


\title{
Model Reduction studies in LQG optimal control design for high-speed tilting railway carriages
}

\author{
Argyrios Zolotas, George Halikias, Roger Goodall and Jun Wang
}

\begin{abstract}
The paper studies the utilisation of model reduction techniques, both physical-based and mathematicalbased, in designing simplified LQG optimal tilt controllers to improve the curving performance of railway coaches at increased running speed. The schemes make exclusive use of local practical signal measurements, i.e. sensors mounted on the current passenger coach. The fundamental problem related with straightforward classical nulling-feedback control is presented, while the commercially-used command-driven with precedence scheme is introduced. A combination of simulation results and, a recently proposed, tilt control system assessment method are employed for assessing the performance of the designed LQG controller.
\end{abstract}

\section{INTRODUCTION}

\section{A. Tilting trains}

Active tilting has become well established in modern railway vehicle technology, with most current high-speed train services in Europe now fitted with tilt and an increasing interest for regional express trains[1]. The concept of tilt is rather straightforward: reduce the lateral acceleration experienced by the passengers, by leaning the bodies of the vehicles inwards on curves, thereby enabling higher vehicle speed operation. These were researched in the 1960s and 1970s, developed for production during the 1980s, and increasingly introduced into service operation during the 1990s.

Early tilt control systems were based solely upon localised-per vehicle measurements (Fig.1a), however it proved impossible at the time to get an appropriate combination of straight track and curve transition performance. Interactions between suspension and controller dynamics (with the sensors being within the control loop) led to control limitations and stability problems (from a theoretical point of view the system performance was limited by nonminimum phase zeros). Since then, tilt controllers have evolved in an incremental sense, the end result of which is a control structure which is not optimised from a system point of view. The industrial norm nowadays is to utilise precedence control [1] devised in the early 1980s as part of the Advanced Passenger Train development [2]. In this scheme a bogie-mounted accelerometer from the vehicle in front is used to provide "precedence" (à priori track

A. Zolotas and R. Goodall are with the Department of Electronic and Electrical Engineering, Loughborough University, Loughborough, Leicestershire LE11 3TU, UK; \{a.c.zolotas, r.m.goodall\}@lboro.ac.uk

G. Halikias is with the School of Engineering and Mathematical Sciences, City University, London EC1V 0HB, UK; g.halikias@city.ac.uk

J. Wang is with the Department of Automation, Tsinghua University, Beijing 100084, P. R. China; junwang@tsinghua. edu.cn information) (via appropriate inter-vehicle cable/signalling connections), carefully designed so that the delay introduced by the filter compensates for the preview time corresponding to approximately a vehicle length (Fig.1b).

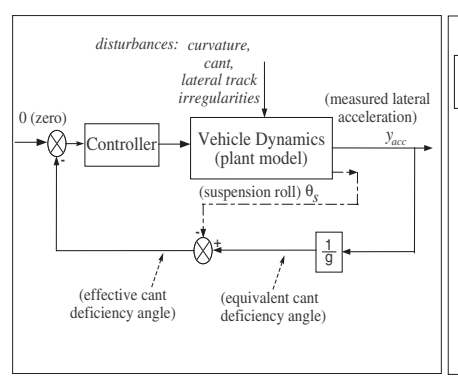

(a) Intuitive partial nulling control (early-type)

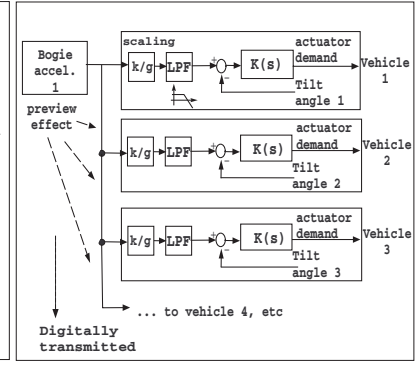

(b) Command driven with precedence control (commercial)
Fig. 1. Tilt control schemes

Nevertheless achieving a satisfactory local tilt control strategy remains an important research topic because of the system simplifications and more straightforward failure detection.

\section{B. Model-reduction methods}

Model reduction techniques attempt to approximate the dynamic model of the plant by a lower-order system which is easier to control. The problem is especially accute for complex or distributed systems, modelled increasingly with the help of sophisticated finite-element or advanced-dynamics software packages.

Reducing the complexity of the system can offer some clear advantages, e.g related to simplification of the design process and the accompanying simulations, elimination of system modes that are irrelevant to control, identification of crucial characteristics of the system, etc. The main motivation of model reduction, however, comes from the increasing application of observer-based modern controldesign methodologies (LQG, $\mathscr{H}_{\infty}$ ). These methods typically result in controllers of an order comparable to that of the plant (possibly augmented with extra filter dynamics). Since a high-order controller is clearly impractical in most situations, some approximation or model reduction techniques is essential.

Two main approaches exist to the model reduction problem. The first, attempts to approximate the input-output characteristics of the plant by a lower order dynamic system, which automatically results in lower-order controllers 
when modern-control methods are employed. Of course the approximation should be carried out sensibly, so that the critical modes of the system are not highly affected. For this reason, most model-reduction techniques using this approach are normally accompanied by some form of robust controldesign methodology (LQG/LTR, $\mathscr{C}_{\infty} / \mu$ ) to ensure tha additional uncertainty introduced by the approximation on system has minimal effects on system stability and performance. The second approach to model reduction attempts to apply approximation techniques directly to the (high-order) controller. Controller order reduction [7] may be viewed as a frequencyweighted problem, emphasizing the approximation in critical frequency ranges for the closed-loop system, e.g. near the cross-over frequency. In this work, only the more traditional approach of plant approximation is considered.

An important recent development in the area of model reduction was the emergence of the Hankel-norm approach, which offers tight error bounds on the infinity-norm of the approximation error. In its various forms (balancedtruncation, Hankel-norm optimal, relative-error, frequencyweighted approximation, etc [7], [10]) it has resulted in algorithms which can be used effectively for small and mediumsize systems. For large-scale problems these methods are not appropriate and Krylov Subspace based methods are preferable [9].

Despite their success, analytical-based model reduction techniques suffer from the disadvantage that the internal description of the reduced model does not relate in an obvious way to the physical variables which typically describe the original (high-order) system. This can be problematic for many engineers who rely on the intuition offered by physical variables to understand and control dynamic systems. In this paper, we attempt to partially bridge the gap between physical and analytical models arising by reducing a high-order model, in the context of a case study in the area of tilt-control of railway vehicles. A set of reduced-order models of this system, arising both from simplifying physical assumptions and analytical reduction techniques are developed, compared and used for optimal control design.

From the area of analytic model reduction, although various methods exist, we concentrate on those methods which retain certain characteristics (modal frequencies) of the original system, and thus easily relate to engineering intuition. This paper concentrates on model reduction, which involves system decomposition in slow and fast modes and in particular its extension presented in [11] via the solution of an optimal Hankel-norm approximation problem with modal constraints.

\section{VEHICLE MODELLING}

The modelling of the baseline railway vehicle is based upon a linearised end-view diagram (Fig.2), including both lateral and roll dynamics of both the body and the bogie plus the contribution of the airspring, the dynamics of the actuation system and the bogie lateral kinematics (resulting to a $13^{\text {th }}$ order model overall).

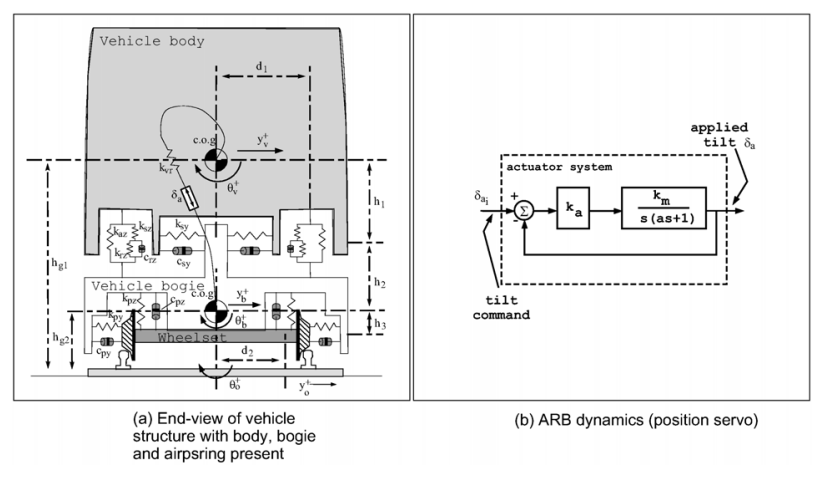

Fig. 2. Tilting vehicle end-view diagram with actuation system

The pair of linear airsprings represents the vertical suspensions, which only contribute to the roll motion of the vehicle (vertical degrees of freedom are ignored). The model also contains the stiffness of an anti-roll bar connected between the body and the bogie frame. Detailed wheelset dynamics were not included for simplicity, however the associated effects are incorporated in the model by using an appropriate 2nd order LP filter (bogie lateral kinematics). The filter was characterised by a $5 \mathrm{~Hz}$ cut-off frequency and $20 \%$ damping.

Active tilt is provided via a rotational displacement actuator, in series with the roll stiffness ('active anti-roll bar (ARB)' [4]), represented by a position servo in series with the ARB; the parameters were chosen to provide $3.5 \mathrm{~Hz}$ bandwidth and 50\% damping. The ARB-system is assumed to provide up to a maximum tilt angle of 10 degrees. The advantages of active ARBs results from their relative simplicity, i.e. small weight increase, low cost, easily fitted as an optional extra to build or as a retro-fit.

The mathematical models of increasing complexity, were developed to encapsulate the lateral and roll dynamics of the tilting vehicle system. These can be represented in the usual state-space form with the state vector $x$ given by

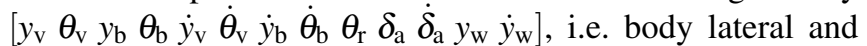
roll position, bogie lateral and roll position, body lateral and roll rate, bogie lateral and roll rate, airspring roll position, applied tilt and tilt rate, bogie kinematics position and rate, respectively. It is worth noting that the system is excited by the track disturbance, including both deterministic (low frequency) and stochastic (higher frequency) elements, while the control input is the ideal tilt command. More details on modelling can be found in [5] and [3].

Substantial coupling exists between the lateral and roll motions which result in two sway modes combining both lateral and roll movement. The 'upper sway' mode, its node appears above the body c.o.g., with predominantly roll movement; and the 'lower sway' mode, node located below the body c.o.g., characterised mainly by lateral motion. The modal analysis of the vehicle is shown in Table I.

\section{Model REDUCTION OF VEHICLE DYNAMICS}

\section{A. Physically-based approximation (PBA)}

The simplest approach to reduce the size of the vehicle model in Section II is to assume a rigid bogie connection to 
TABLE I

$13^{\text {th }}$ ORDER ARB VEHICLE MODAL ANALYSIS

\begin{tabular}{|l||r||r|}
\hline Mode & Damping (\%) & Frequency (Hz) \\
\hline \hline Body lower sway & 16.5 & 0.67 \\
\hline Body upper sway & 27.2 & 1.50 \\
\hline Bogie lateral & 12.4 & 26.80 \\
\hline Bogie roll & 20.8 & 11.10 \\
\hline Bogie kinematics & 20.0 & 5.00 \\
\hline Actuation system & 50.0 & 3.50 \\
\hline Airspring mode & 100.0 & 3.70 \\
\hline
\end{tabular}

the rail track, i.e. removal of bogie dynamics and kinematics (thus the track inputs have a direct effect on the secondary suspensions). Also to assume an ideal actuator, thus removing the actuation dynamics. Two, physically-reduced, model cases are considered: (PBA1) keep body states plus airspring state $\left(5^{\text {th }}\right.$ order) and (PBA2) keep body states only $\left(4^{\text {th }}\right.$ order). Note that the structure of the reduced states has been retained from the baseline model, i.e. Table II shows the

TABLE II

DYNAMIC MODES OF PHYSICALLY-BASED REDUCED ORDER MODELS WITH SYSTEM STATES: (PBA1) $\left[y_{\mathrm{v}} \theta_{\mathrm{v}} \dot{y}_{\mathrm{v}} \dot{\theta}_{\mathrm{v}} \theta_{\mathrm{r}}\right]$, (PBA2) $\left[y_{\mathrm{v}} \theta_{\mathrm{v}} \dot{y}_{\mathrm{v}} \dot{\theta}_{\mathrm{v}}\right]$

\begin{tabular}{|c||r|r||r|r|}
\hline \multirow{2}{*}{\multicolumn{1}{|c||}{ Mode }} & \multicolumn{2}{c||}{ model (PBA1) } & \multicolumn{2}{c|}{ model(PBA2) } \\
\cline { 2 - 5 } & $\zeta(\%)$ & $\omega(\mathrm{Hz})$ & $\zeta(\%)$ & $\omega(\mathrm{Hz})$ \\
\hline \hline (Body) lower sway & 24.0 & 0.75 & 25.8 & 0.78 \\
\hline (Body) upper sway & 19.6 & 1.78 & 12.8 & 1.93 \\
\hline (Airspring) mode & 100.0 & 4.00 & $\mathrm{n} / \mathrm{a}$ & $\mathrm{n} / \mathrm{a}$ \\
\hline
\end{tabular}

modes of the reduced models, which appear at around the same frequencies, although with changes in the damping (especially for model 'PBA2' where no airspring exists the damping for the upper sway mode is further reduced, as one expects).

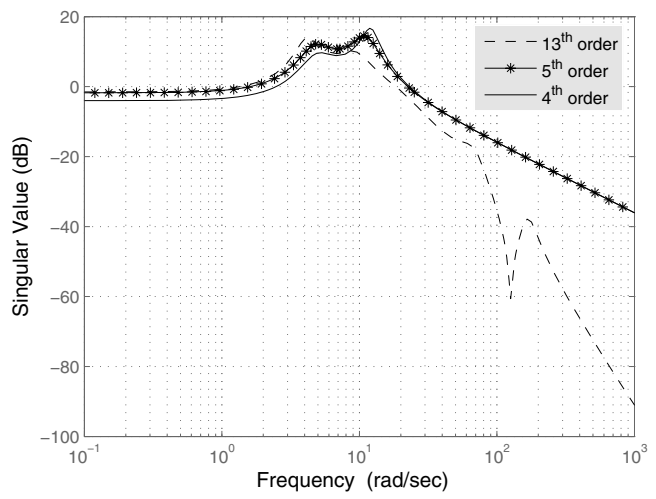

Fig. 3. Singular value plot for physically-based reduction

Fig.3 illustrates the singular value plot of a sample SISO TF from actuator angle $\left(u=\delta_{a_{i}}\right)$ to the effective cant deficiency for $60 \%$ partial tilt $\theta_{e c d}$ (see Fig.1(a)). It can be seen that both $5^{\text {th }}$ and $4^{\text {th }}$ reduced order models are quite close, their modelling error (with respect to the $13^{\text {th }}$ order model) increases significantly at high frequency. The actuator dynamics could be further included in both reduced models to provide an extra $-40 \mathrm{~dB} /$ decade roll-off at high frequencies (of course at the expense of 2 extra states, i.e. $\delta_{a}, \dot{\delta}_{a}$ ) if necessary; thus providing better approximation of the original features at those frequencies. Note that all disturbance signals have been set to zero for the reduction procedures in Section III.

\section{B. Analytically-based reduction}

1) Slow-fast model decomposition: In this approach the system is decomposed into slow and fast modes, whereby the former is retained and the latter eliminated. The method [12] is briefly described here. Let the original system be given in state-space form as

$$
\dot{x}(t)=A x(t)+B u(t), y(t)=C x(t)+D u(t)
$$

with $A$ stable. Introduce an orthogonal state-space transformation $V$ so that $V^{T} A V$ is in real-Schur form. In this form, the structure of the transformed matrix is essentially upper triangular, with real eigenvalues appearing on the main diagonal, while (simple) complex-conjugate eigenvalues correspond to $2 \times 2$ blocks extending above and below the main diagonal. The eigenvalues (assumed stable) are ordered according to their magnitude, so that the slow modes are located in the upper-diagonal block. Thus, if $m$ represents the number of slow modes that we wish to retain,

$$
\left(\begin{array}{l}
V_{1}^{T} \\
V_{2}^{T}
\end{array}\right) A\left(\begin{array}{ll}
V_{1} & V_{2}
\end{array}\right)=\left(\begin{array}{cc}
A_{s} & A_{12} \\
0 & A_{f}
\end{array}\right)
$$

where $A_{s}$ and $A_{f}$ denotes the two blocks containing the slow and fast modes, respectively, and where $V_{1}$ consists of the first $m$ columns of $V$. Note that (simple) complex conjugate eigenvalues are not allowed to be split. Next, let $X$ be the solution of the Sylvester equation

$$
A_{s} X-X A_{f}+A_{12}=0
$$

It is well-known that the solution to this equation exists and is unique, provided that $\lambda_{i}\left(A_{s}\right)-\bar{\lambda}_{j}\left(A_{f}\right) \neq 0$, for all possible $i$ and $j$; note that this is automatically satisfied if the eigenvalues are separated by a positive gap in magnitude as is assumed here. Introducing the additional transformation

$$
\left(\begin{array}{cc}
I & -X \\
0 & I
\end{array}\right)\left(\begin{array}{l}
V_{1}^{T} \\
V_{2}^{T}
\end{array}\right) A\left(\begin{array}{ll}
V_{1} & V_{2}
\end{array}\right)\left(\begin{array}{cc}
I & X \\
0 & I
\end{array}\right)=\left(\begin{array}{cc}
A_{s} & 0 \\
0 & A_{f}
\end{array}\right)
$$

which allows the separation of slow and fast modes via parallel decomposition. Note that the state vector, in the new coordinate system, is related to $x$ via the transformation

$$
z(t)=\left(\begin{array}{c}
z_{1}(t) \\
z_{2}(t)
\end{array}\right)=\left(\begin{array}{c}
V_{1}^{T}-X V_{2}^{T} \\
V_{2}^{T}
\end{array}\right) x(t)
$$

where $z_{1}(t)$ is the state-vector of the slow part of the realization. This allows us to retain the physical significance of the new state variables $z_{i}$, through their link with the original state-vector $x$; this link could be further developed for LQR-based controller designs. 
2) Modal Hankel-norm approximation: A natural extension of the slow-fast decomposition method outlined above is the method described in [11], which relates to formulating and solving a constrained optimal Hankel-norm approximation problem with modal constraints. The original system $G(s)$ (assumed stable) is split additively into two systems $G_{1}(s)$ and $G_{2}(s)$, i.e. taken to represent the slow and fast parts of $G(s) . G_{1}(s)$ (the slow part) is approximated by a system of the same degree, $\hat{G}_{1}(s)$, which shares its $A$ and $C$ matrix with $G_{1}(s)$ (in the SISO case $\hat{G}_{1}(s)$ is constrained to have the same poles as $G_{1}(s)$, although not necessarily the same zeros); $G_{2}(s)$ is approximated freely by a reducedorder model $\hat{G}_{2}(s)$. The overall problem is to choose $\hat{G}_{1}(s)$ and $\hat{G}_{2}(s)$ (under the above constraints), so that the Hankel norm of the approximation error $\left\|G(s)-\hat{G}_{1}(s)-\hat{G}_{2}(s)\right\|_{H}$ is minimised. The problem is solved via a left-coprime factorisation (with inner denominator) and the solution of a (weighted) optimal Hankel-norm approximation problem (for more details see [11]).

The method was applied to the full-order model (actuator angle $u=\delta_{a_{i}}$ to effective cant deficiency for $60 \%$ tilt compensation on steady curve $\theta_{e c d}$ ) (all disturbance signals set to zero). The slow part of the system (4 states) corresponding to the two body sway modes was retained, while the faster part (9 states) was approximated via systems of varying degree $k$ where $k \leq 9$. A sample Bode plot of both the original and reduced order systems for $k=1,3,5$ is shown in Fig.4

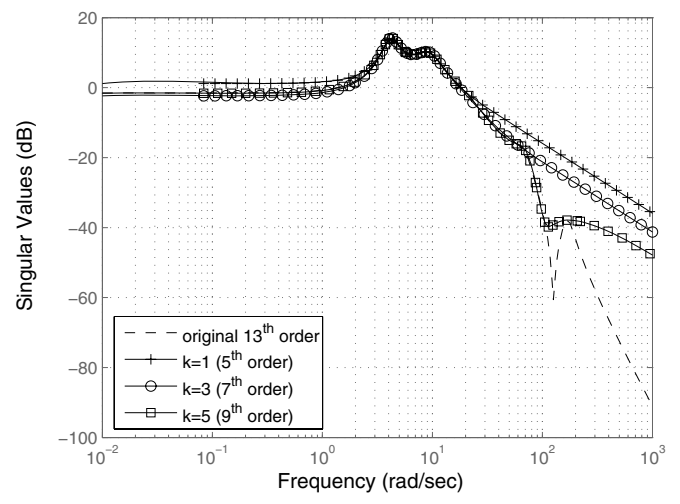

Fig. 4. Singular value plot of Modal Hankel-norm approx. for $k=1,3,5$

As seen from the figure, the procedure successfully retains the slow modes and approximates the fast modes directly via an appropriate filter. The approximation for $k=3$, i.e. $7^{\text {th }}$ order design model, is a rather promising choice for subsequent control design.

\section{Tilt Control Requirements AND Assessment APPROACH}

\section{A. Requirements}

The performance of tilt control systems on the curve transitions is critical; most importantly the passenger ride comfort provided by the tilting vehicle should not be (significantly) degraded compared to the non-tilting vehicle speeds.
The main objectives of any tilt control system are: (i) to provide an acceptably fast response to changes in track cant and curvature (deterministic track features), and (ii) not to react significantly to track irregularities (stochastic track features). However, any tilt control system directly controls the secondary suspension roll angle and not the vehicle lateral acceleration. Hence, there is a fundamental trade-off between the vehicle curve transition response and straight track performance. Moreover, for reasons of human perception, designers utilise partial tilt compensation. In such a case the passenger will still experience a small amount of acceleration on steady curve, in order to minimise motion sickness phenomena.

From a control design point of view the objectives of the tilt system can be translated as: increasing the response of the system at low frequencies (deterministic track features), while reducing the high frequency system response (stochastic track features) and maintaining stability.

\section{B. Assessment}

Two main design criteria are concerned with tilting trains: (i) providing a fast response on curved track (deterministic criterion), (ii) maintain a good ride quality in response to track irregularities on straight track (stochastic criterion).

The assessment of the curve transition is based upon the idea of "ideal tilting", i.e. where the tilt action follows the specified tilt compensation in an ideal manner according to the maximum tilt angle and cant deficiency compensation factor. Deviations from the "ideal tilting" response (related to tilt angle, roll velocity and lateral acceleration) quantifies the additional dynamic effects which are caused by the suspension/controller dynamics on the transitions to and from the curves, and provides an objective measure which can be used to compare different strategies. Note that the calculation of $P_{C T}$ factors, a comprehensive experimental/empirical study which provides the percentage of (both standing and seated) passengers who feel uncomfortable during the curve transition, is also included in this stage [6].

The straight track performance criterion is to allow a degradation of the lateral ride quality by no more than a specified margin compared with the non-tilting response, a typical value being $7.5 \%$. It is essential, for assessing the tilt controller performance, this comparison to be made at the higher (tilting) speed.

\section{LQG CONTROL SYNTHESIS}

In this section we utilise the reduced $7^{\text {th }}$ order SISO model from section III-B.2 (from ideal actuator roll input $u=\delta_{a_{i}}$ to effective cant deficiency $\theta_{e c d}$ output), for Linear Quadratic Gaussian (LQG) control design and assess its effectiveness when incorporated to the original model. Linear Quadratic Gaussian control is a well documented in [8] and thus we omit its theoretical details.

\section{A. Tilt Control Synthesis}

Recall that the reduced order model for control has been derived with all disturbance signals set to zero, and also its 
states are a linear combination of the original states. As a result it is not as straightforward to base the control design strictly on the original (real) aspects of the problem. Note also that the LQG SISO design is a simple extension of the conventional (classical) nulling tilt problem in an optimal control framework.

The view adopted here is to synthesize the tilt controller via LQG theory with the weighting matrices $Q, R, W, V$ (state and control weights, and process and sensor noise covariances respectively) purely considered as tuning parameters until an appropriate design is obtained. In particular, the structure of the LQG tilt compensator is found by shaping the principal gains of the system given by,

$$
\dot{x}=A x+B u+\Gamma w, y=C x+v
$$

First the Kalman Filter is designed via tuning $W, V$ to obtain a satisfactory return ratio $-C(s I-A)^{-1} K_{f}\left(K_{f}\right.$ is the Kalman gain) at the plant output, while the LQR is synthesized via tuning $Q, R$ such that the return ratio at the output of the compensated plant converges sufficiently close to $-C(s I-A)^{-1} K_{f}$ over the frequency range of interest (Loop Transfer Recovery-LTR). Note that for correct tilt compensation on steady curve the LQG compensator should incorporate integral action (thus providing zero sensitivity at zero frequencies). Thus, the augmented system is

$$
\left[\begin{array}{l}
\dot{x} \\
\dot{\xi}
\end{array}\right]=\left[\begin{array}{cc}
A & \Gamma \\
0 & A_{w}
\end{array}\right]\left[\begin{array}{l}
x \\
\xi
\end{array}\right]+\left[\begin{array}{ll}
B & 0 \\
0 & 1
\end{array}\right]\left[\begin{array}{l}
u \\
\tilde{w}
\end{array}\right], y=\left[\begin{array}{ll}
C & 0
\end{array}\right]\left[\begin{array}{l}
x \\
\xi
\end{array}\right]+v
$$

with the process noise $w$ (from (6)) being the integral $\xi$ of the virtual process noise input $\tilde{w}$ (with covariance $\tilde{W}$ ). The eigenvalues of $A_{w}$ were placed just to the left of the origin for the solution to exist (in the implementation stage these were moved back to the origin for proper integration).

We start with the simplest possible choices for $\Gamma, V$ equal to $B, 1$ respectively (still for the SISO model). $\Gamma=B$ refers to any (virtual) disturbances on the plant acting via the input, rather than the actual track disturbances from the original model. $\tilde{W}$ is adjusted accordingly to improve (shape) the output return ratio. Fig.5 illustrates the return ratio $-C(s I-A)^{-1} K_{f}$ for various $\tilde{W}$.

The return ratio of the Kalman Filter for $\tilde{W}=10$ with a crossover of approx 10rad/s, is a good choice for recovery. However a simple calculation of the transmission zeros for the design plant reveals a non-minimum phase zero at approximately $5.5 \mathrm{rad} / \mathrm{s}$ (this is characteristic for such a setup in tilting trains [3]), thus making full recovery difficult. For illustration, we follow the usual LTR procedure up to the limit of recovery allowed from the nonminimum phase zero (usually the achievable bandwidth of the system is less than half of the RHP zero frequency [8]).

The design of $\mathrm{LQR}$ is based upon choosing $R=1$ and $Q=$ $C^{T} C+q I$, where $C^{T} C$ relates to the weighting of the reduced order states from the effective cant deficiency output (this has retained information from the original model) and $q I$ is the additional diagonal weighting relative to the reduced-order set of states. Fig. 6 illustrates the amount of recovery at plant

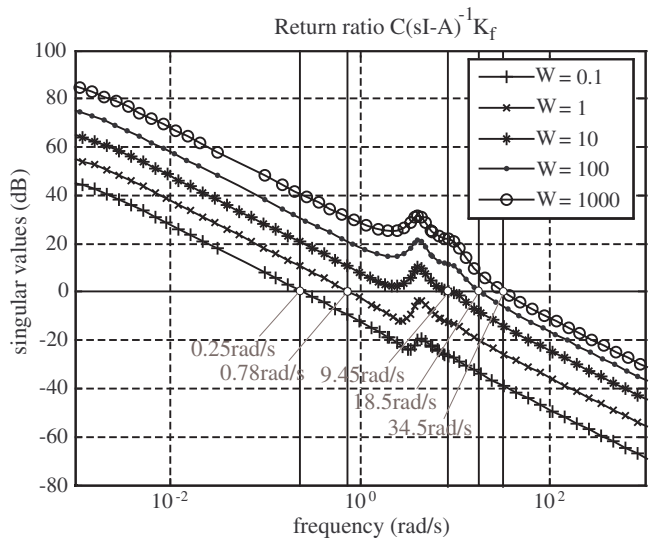

Fig. 5. Kalman Filter return ratio for various $W$

LTR @ plant output

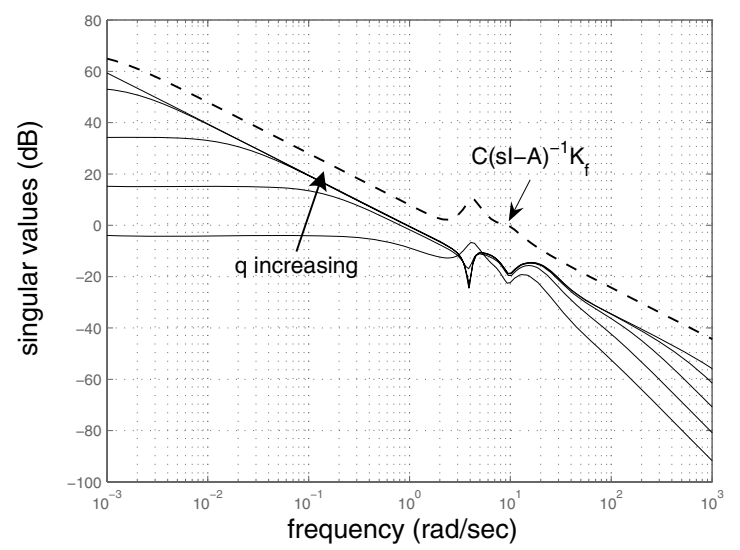

Fig. 6. LTR at plant output for increasing $q=0.1,1,10,100,1000$

output for increasing values of $q$, where in fact there is no point in recovering after $q=100$ (the actual crossover limit is placed by the nonminimum phase zero). The synthesized ( $8^{\text {th }}$ order) LQG controller realization is given by

$$
K_{l q g} \stackrel{s}{=}\left[\begin{array}{c|c}
\bar{A}-\bar{B} K_{r}-K_{f} \bar{C} & K_{f} \\
\hline-K_{r} & 0
\end{array}\right]
$$

with $K_{r}$ the LQR gain matrix, $\bar{B}=\left[\begin{array}{ll}B & 0\end{array}\right]^{T}$. This was reduced further to a $6^{\text {th }}$ order controller (via Balanced Truncation) without significant degradation in performance. The sensitivity of the system together with the principal gains of the LQG controller can be seen in Fig.7. Incorporating the controller in the original system results to a approximately the same sensitivity (illustrating the effectiveness of model reduction) compared to the reduced design plant. The principal gain plot of the LQG controller clearly shows its integral action.

The time domain results for the lateral acceleration felt by the passengers can be seen in Fig. 8. It illustrates the ideal acceleration (assuming ideal tilt behaviour), the LQGbased and a classical nulling-PI scheme [3]. The deterministic track input used consists of a curved section with a radius of $1000 \mathrm{~m}$ superimposed by a maximum track cant angle of $155 \mathrm{~mm}\left(6^{\circ}\right)$, with a tilting speed of $209 \mathrm{~km} / \mathrm{h}$. The 
TABLE III

TILT PERFORMANCE ASSESSMENT @ 209(KM/H)

\begin{tabular}{llrrl}
\hline \multicolumn{2}{c}{ DETERMINISTIC (CURVE TRANSITION) } & SISO LQG-BASED & CLASSICAL-PI & \\
\hline Lateral accel. & - steady-state & 9.53 & $\mathrm{n} / \mathrm{a}$ & $(\% \mathrm{~g})$ \\
(actual vs ideal) & - R.M.S. deviation error & 4.0 & 5.4 & $(\% \mathrm{~g})$ \\
& - peak value & 16.7 & 19.3 & $(\% \mathrm{~g})$ \\
Roll velocity & - R.M.S. deviation & 0.033 & 0.032 & $(\mathrm{rad} / \mathrm{s})$ \\
& - peak value & 0.102 & 0.087 & $(\mathrm{rad} / \mathrm{s})$ \\
& - peak jerk level & 8.7 & 10.2 & $(\% \mathrm{~g} / \mathrm{s})$ \\
$P_{C T} /$ P-factor & - standing & 64.0 & 71.0 & $(\%$ of passengers) \\
& - seated & 19.3 & 22.5 & (\% of passengers) \\
\hline \multicolumn{2}{c}{ STOCHASTIC (STRAIGHT TRACK) } & & & \\
\hline Passenger comfort & - R.M.S. passive (equiv.) & 3.78 & 3.78 & $(\% \mathrm{~g})$ \\
& - R.M.S. active & 4.1 & 4.13 & $(\% \mathrm{~g})$ \\
& - degradation & 5.67 & 6.17 & $(\%)$ \\
\hline
\end{tabular}

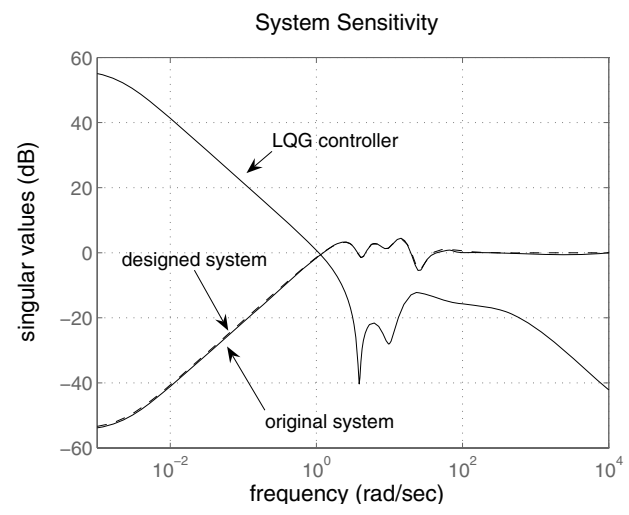

Fig. 7. System Sensitivity $(I-G K)^{-1}$ system with LQG controller

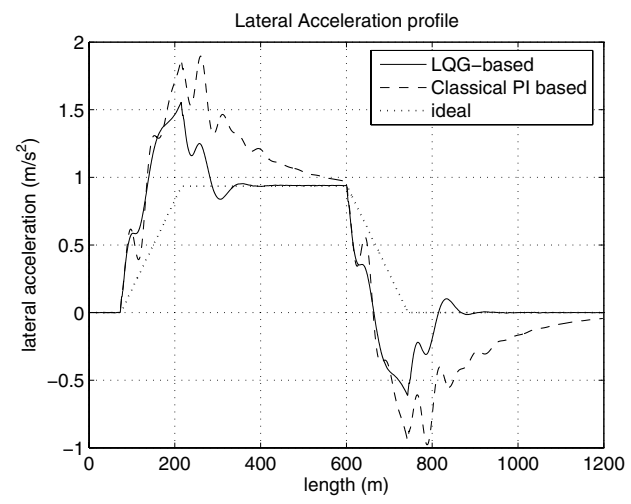

Fig. 8. Lateral acceleration profile on curved track

performance assessment of the controller (based on section IV-B) is presented in Table III. Although the LQG-based is a straightforward optimal extension of the classical nulling scheme, the performance is much improved (emphasizing robustness with the additional damping injected).

\section{CONCLUSIONS AND FUTURE WORK}

The paper discussed on model reduction issues in designing optimal controllers for tilting railway vehicles. The design is based on practical measurements using local vehicle information, i.e. with no 'a priori' information on track profile. The optimal nulling-type SISO LQG controller based on the reduced order model of the tilting vehicle, offers a significant improvement of tilting performance compared to the classical nulling-equivalent although both schemes are based on the same concept (and having the same limitations). Shaping the principal gains of the return ratio of the system with the automatic LQG procedure, avoids manually designing networks of classical compensators. In addition the LQG-scheme provides improved robustness properties, although designed on the reduced-order system. Future work is concentrated on the re-formulation of the scheme with extra sensor information and process noise choices and further controller reduction in closed loop. The paper should be of considerable interest to control engineers who have to provide practical solutions but may be put off by the potential complexity of normal model-based control techniques.

\section{REFERENCES}

[1] R.M. Goodall, Tilting Trains and Beyond - the Future for Active Railway Suspensions: Part 1 Improving passenger comfort, Computing and Control Engineering Journal IEE, vol. August, 1999, pp153-160.

[2] D. Boocock and B.L. King, The Development of the Prototype Advanced Passenger Train, Proceedings, Institution of Mechanical Engineers,vol. 196, March, 1982, pp. 35-46.

[3] A.C. Zolotas, Advanced Control Strategies for Tilting Trains, $\mathrm{PhD}$ Thesis, Loughborough University, 2002.

[4] J.T. Pearson, R.M. Goodall and I. Pratt, Control System Studies of an Active Anti-Roll Bar Tilt System for Railway Vehicles, Proceedings, Inst. of Mechanical Engineers, vol. 212, number F1, 1998, pp. 43-60.

[5] A.C. Zolotas and R.M. Goodall, Advanced Control Strategies For Tilting Railway Vehicles, In Proceedings UKACC Control 2000, Cambridge UK, September, 2000.

[6] R.M. Goodall, A.C. Zolotas and J. Evans, Assessment of the Performance of Tilt System Controllers, Proc of the Railway Technology Conference IMechE, no. C580/028/2000, Birmingham, UK, November, 2000, pp 231-239.

[7] K. Zhou and J. C. Doyle, Essentials of Robust Control, Prentice-Hall, 1998

[8] S. Skogestad and I. Postlethwaite, Multivariable Feedback Control: Analysis and Desgn, Wiley, 2000.

[9] I.M. Jaimoukha and E. M. Kasenally , Implicitly Restarted Krylov Subspace Methods for Stable Partial Realizations, SIAM J MATRIX ANAL A, 18 (3),pp. 633-652, July 1997.

[10] A.C. Antoulas, D.C. Sorensen, and S. Gugercin, Structured Matrices in Operator Theory, Numerical Analysis, Control, Signal and Image Processing, Contemporary Mathematics, AMS publications, 2001.

[11] Y.S. Hung and M.A. Muzlifah, Hankel-norm model reduction with fixed modes, IEEE Trans on Automatic Control, Vol. 35 , Issue 3, pp. 373-377, 1990

[12] M. G. Safonov, E. A. Jonckheere, M. Verma and D. J. N. Limebeer, Synthesis of Positive Real Multivariable Feedback Systems, Int. J. Control, vol. 45, no. 3, pp. 817-842, 1987. 